\title{
BMJ Open Exogenous sex steroid hormones and asthma in females: protocol for a population-based retrospective cohort study using a UK primary care database
}

\author{
Bright I Nwaru, ${ }^{1,2,3}$ Colin R Simpson, ${ }^{3}$ Ireneous N Soyiri, ${ }^{3}$ Rebecca Pillinger, ${ }^{3}$ \\ Francis Appiagyei, ${ }^{4}$ Dermot Ryan, ${ }^{3,4}$ Hilary Critchley, ${ }^{5}$ David B Price,,${ }^{4,6,7}$ \\ Catherine M Hawrylowicz, ${ }^{8}$ Aziz Sheikh ${ }^{3}$
}

To cite: Nwaru BI, Simpson CR, Soyiri IN, et al. Exogenous sex steroid hormones and asthma in females: protocol for a population-based retrospective cohort study using a UK primary care database. BMJ Open 2018;8:e020075. doi:10.1136/ bmjopen-2017-020075

- Prepublication history for this paper is available online. To view these files, please visit the journal online (http://dx.doi org/10.1136/bmjopen-2017020075).

Received 12 0ctober 2017 Revised 16 May 2018 Accepted 24 May 2018
Check for updates

(C) Author(s) (or their employer(s)) 2018. Re-use permitted under CC BY-NC. No commercial re-use. See rights and permissions. Published by BMJ.

For numbered affiliations see end of article.

Correspondence to

Dr Bright I Nwaru;

bright.nwaru@gu.se

\section{ABSTRACT}

Introduction Female sex steroid hormones have been implicated in sex-related differences in the development and clinical outcomes of asthma. The role of exogenous sex steroids, however, remains unclear. Our recent systematic review highlighted the lack of high-quality populationbased studies investigating this subject. We aim to investigate whether the use of hormonal contraception and hormone replacement therapy (HRT), subtypes and route of administration are associated with asthma onset and clinical outcomes in reproductive age and perimenopausal/ postmenopausal females.

Methods and analysis Using the Optimum Patient Care Research Database (OPCRD), a national primary care database in the UK, we will construct a retrospective longitudinal cohort of reproductive age (16-45 years) and perimenopausal/postmenopausal (46-70 years) females. We will estimate the risk of new-onset asthma using Cox regression and multilevel modelling for repeated asthma outcomes, such as asthma attacks. We will adjust for confounding factors in all analyses. We will evaluate interactions between the use of exogenous sex hormones and body mass index and smoking by calculating the relative excess risk due to interaction and the attributable proportion due to interaction. With $90 \%$ power, we need 23700 reproductive age females to detect a $20 \%$ reduction (risk ratio 0.8 ) in asthma attacks for use of any hormonal contraception and 6000 perimenopausal/postmenopausal females to detect a $40 \%$ (risk ratio 1.40) increased risk of asthma attacks for use of any HRT.

Ethics and dissemination We have obtained approval (ADEPT1317) from the Anonymised Data Ethics and Protocol Transparency Committee which grants project-specific ethics approvals for the use of OPCRD data. Optimum Patient Care has an existing NHS Health Research Authority ethics approval for the use of OPCRD data for research (15/EM/150). We will present our findings at national and international scientific meetings and publish the results in international peerreviewed journals.

Trial registration number EUPAS22967.

\section{INTRODUCTION}

Asthma is more common in boys than girls during early childhood. ${ }^{1-3}$ However, after
Strengths and limitations of this study

- The longitudinal nature of this study will fill an important gap in the evidence base as there is a paucity of longitudinal studies investigating the role of exogenous sex steroid hormones in asthma in females.

- As a study based on primary care database, both exposure and outcome measures will be objectively defined.

- This study is based on the general and unselected population; therefore, our findings will be generalisable to the general population of reproductive age and perimenopausal/postmenopausal females.

- Overall, this study will inform the direction for further mechanistic and interventional works.

- A limitation of this work is that we will define reproductive and menopausal status of females based only on the ages of women which is the only information available from the General Practitioner database for this purpose.

puberty, the prevalence and severity of asthma are higher in females than males. ${ }^{1-3}$ Female sex steroid hormones are believed, at least in part, to explain these sex-related variations in asthma outcomes. ${ }^{1-3}$ Variations in asthma incidence and clinical outcomes are seen to follow the hormonal transitional points in the female reproductive life course, in particular, puberty, menarche, menstruation, pregnancy and menopause. ${ }^{4}$ Fluctuations in oestradiol and progesterone levels during the menstrual cycle have been linked to worsening of asthma symptoms in females, ${ }^{5}$ although immunological mechanisms underlying these effects have not been clearly defined. In comparison to non-menopausal females, transitional menopausal females and both early and late postmenopausal females were at increased risk of new-onset asthma. In primary studies, 
the role of menopause on risk of current symptoms of asthma were equivocal, ${ }^{6}$ but a recent meta-analysis of these studies showed a moderately increased risk. ${ }^{7}$

Both oestrogen and progesterone influence smooth muscle functions, inflammation and airway responsiveness. ${ }^{8-10}$ Studies in animal models indicate that oestrogen increases T-helper (Th) 2 cell responses associated with allergic asthma. ${ }^{10}$ A recent study showed that in patients with severe asthma, both oestrogen and progesterone were associated with a decrease in the expression of the left-7f microRNA and an increase in interleukin (IL)-23/IL-23 receptor signalling and IL-17A production, an effect that was more marked in women versus men. ${ }^{11}$ Some evidence suggests that external suppression of endogenous sex steroid production through the use of exogenous hormonal contraception may improve asthma outcomes, ${ }^{12-17}$ whereas the use of hormone replacement therapy (HRT) by menopausal women may increase the risk of new-onset asthma and risk of poor clinical outcomes of asthma. ${ }^{18-23}$ Females with asthma exhibit enhanced Th17 responses, reduced asthma symptoms and improved lung function when using hormonal contraception. 59102425

The role of use of hormonal contraceptives both in the development of new-onset asthma ${ }^{2326}$ and manifestation of current symptoms of asthma ${ }^{16}$ has been conflicting. Using the serial cross-sectional Scottish Health Surveys, we recently observed substantial reductions in asthma exacerbations and hospital episodes in females using hormonal contraception compared with those not using hormonal contraception. ${ }^{15}$ This work was followed by a comprehensive synthesis of the underlying evidence which revealed inherent methodological limitations in previous studies on the topic, including a paucity of prospective longitudinal studies and limitations in the measurement of sex steroids and asthma outcomes. ${ }^{7}$ To overcome these weaknesses and thus clarify whether the role of sex steroid hormones in asthma in females is causal, well-designed long-term longitudinal studies with well-characterised populations are required. We plan to investigate the role of exogenous sex steroid hormones in the development of asthma and manifestation of clinical and patient-reported outcomes in females by creating a retrospective longitudinal cohort of reproductive age and perimenopausal/postmenopausal females using the Optimum Patient Care Research Database (OPCRD). Specifically, we aim to investigate the:

1. Associations between use of hormonal contraception and asthma onset, attacks, severity, mortality and health-related quality of life (HRQoL) in reproductive-age females.

2. Impact of type of menopause (surgical vs non-surgical) and use of HRT on asthma onset, attacks, severity, mortality and HRQoL in perimenopausal/postmenopausal females.

3. Interactions between exogenous sex hormones, body mass index (BMI), cigarette smoking and alcohol consumption in these associations.
METHODS

\section{Patient and Public Involvement}

In developing the study protocol at the grant application stage, we worked closely with the members of the Patient and Public Involvement (PPI) group of the Asthma UK Centre for Applied Research, University of Edinburgh. Selected members of the PPI helped with the grant application, including commenting and refining the plain English summary of the application, and ensuring that the public perspectives are thoroughly embedded throughout the research plans. We will also invite the PPI group to comment on the findings of the study, play an active part in developing the key messages from the study for public consumption and help in disseminating our findings to the public via website, social media, conferences and public engagement events around the study.

\section{Study design and population}

OPCRD is a bespoke longitudinal deidentified primary care database representing over 600 general practices across the UK and is regularly used to conduct epidemiological, clinical and pharmaceutical research (http:// optimumpatientcare.org/opcrd/). A major advantage of the OPCRD database is the focus on respiratory outcomes; in up to $10 \%$ of patients with asthma, patient-reported questionnaire data on asthma outcomes are available. This provides the opportunity to study both clinical and patient-reported outcomes from the database. The study population for the present investigation will comprise all females aged 16-70years in OPCRD. We will construct two independent cohorts to address the study objectives, namely:

1. Reproductive age females (16-45 years of age) to study the associations between use of hormonal contraception and the study outcomes.

2. Perimenopausal (46-55years)/postmenopausal females (56-70years) to study the associations between type of menopause and HRT and the study outcomes.

\section{Exposures}

We will ascertain the use of hormonal contraception and HRT and type of menopause by means of the Read Clinical Classification System (Read codes). ${ }^{27} 28$ We will define the following exposures:

1. Reproductive age females: use of hormonal contraception versus non-use of hormonal contraception; subtypes (oestrogen/progestogen combined, progestogen only vs non-use of hormonal contraception); route of administration (transdermal, subcutaneous, intramuscular, local intrauterine vs oral route), and frequency and duration of use (as a count exposure).

2. Menopausal status: perimenopausal (46-55years) versus non-menopausal (<46years); postmenopausal (5670 years) versus non-menopausal ( $<46$ years)

3. Perimenopausal/postmenopausal females: use of HRT versus non-HRT use, subtypes (oestrogen/progestogen combined, oestrogen-only vs non-HRT use), route of administration (transdermal, subcutaneous, local 
intrauterine vs oral route), and frequency and duration of use (as a count exposure).

\section{Confounders}

Potential confounders will be extracted from the database using their respective Read codes, including age, parity, BMI, smoking, current use of asthma treatments and level of adherence to these using our definition based on the algorithm developed within the OPCRD, ${ }^{29}$ Index of Multiple Deprivation, ${ }^{30}$ co-morbidity based on the Charlson index ${ }^{31}$ and other indications for use of hormonal contraception (eg, endometriosis, polycystic ovary syndrome (PCOS), menorrhagia, acne and metrorraghia) and HRT (eg, onset of menopause, endometriosis and indicators for hysterectomy for symptomatic uterine fibroids, and heavy menstrual bleeding) besides contraception and symptoms of menopausal transition, respectively. Endometriosis ${ }^{32}$ and PCOS,${ }^{33}$ key indicators for the use of hormonal contraceptives among reproductive age women, have been linked to risk of asthma and, therefore, are important confounders in the association between use of hormonal contraceptives and asthma. For HRT, the onset of menopause itself and hysterectomy, other indicators for use of HRT besides symptoms of menopausal transition, have also been linked to asthma. ${ }^{621}$

\section{Outcomes}

The primary outcomes will include new-onset asthma, asthma attacks and severity. New-onset asthma will be defined as the first General Practitioner (GP)-recorded asthma event (including diagnosis, hospitalisation, medication prescription or any other asthma event) occurring at least 5 years from the start of the follow-up date. We will exclude individuals with a relevant asthma event recorded up to 5 years after the start of follow-up date. We assume that within a 5-year period, a patient with asthma should have had at least one clinical encounter. Asthma attacks and severity will be defined based on the frequency of GP consultation, accident and emergency attendance, oral steroid courses and hospital admissions for asthma. Using these parameters and based on algorithm we have now developed within the OPCRD database, patients will be classified according to the British Thoracic Society and Scottish Intercollegiate Guidelines Network asthma severity steps. ${ }^{34}$ The outcomes will be determined using relevant Read codes. The secondary outcomes will include patient-reported asthma symptoms, medication use and HRQoL. Data on HRQoL will be assessed from patient-completed questionnaires on asthma symptoms and quality of life which is an adjunct data collected from about $10 \%$ of patients and added to the OPCRD database.

\section{Follow-up period}

We will follow the participants from baseline starting from 1 January 2000 or date of registration until 31 December 2016. With regard to onset of asthma, exit date from the cohort will be defined as the date of first diagnosis of asthma (ie, date of first record of an asthma event), death, deregistration from a practice or end of follow-up (31 December 2016), whichever comes first.

\section{Statistical analyses}

Prior to the main analyses, the data will undergo relevant quality checks, including relevant variable categorisation (rescaling where appropriate) and checks for missingness. We will undertake a complete case analysis and also perform multiple imputation for variables with missing values. We will perform 20 imputations in order to enhance the efficiency of the estimates and will use Rubin's rule to combine the estimates across the 20 datasets. ${ }^{35}$ Where the specific time of onset of asthma is observable, we will perform survival analysis using the log-rank test to describe the survival functions of the groups as defined by use of sex hormones. We will use Cox proportional hazards regression to study the associations between exogenous sex hormones and the first record of an asthma event. Multilevel modelling will be used to estimate associations where the outcomes are repeated, for example, number of asthma attacks and medication use. Since the change in hormone levels with contraceptive use is expected to differ between women, random coefficient models will be fitted, so that in turn the relationship between contraception use and asthma outcomes can differ between women. We will undertake analyses incorporating propensity scores using matching (exposed vs unexposed). ${ }^{36}$ The model will be non-parsimonious in order to include a wide range of factors that influence propensity to be prescribed hormonal contraceptives and HRT. To minimise potential biases, we will undertake different scenarios of sensitivity analyses in order to evaluate the robustness of our findings, including analyses for potential selection bias at baseline, unmeasured confounding and information bias. ${ }^{37}$ These sets of bias analyses will be aided by deriving relevant internal data from a subset of the study population where possible. Alternatively, we will obtain external validation data (for instance, from the Secure Anonymised Information Linkage in Wales) that will provide the basis for defining the sensitivities of the different measures and allow appropriate adjustments to be made to our estimates. We will also evaluate the potential of confounding by indication bias by stratifying the analyses by the relevant disease indication for using sex steroid preparations as indicated in the Confounders section. To estimate the potential interactions between sex hormones and BMI and cigarette smoking, we will calculate the relative excess risk due to interaction and the attributable proportion due to interaction. ${ }^{38}$ All estimates will be accompanied by their respective $95 \%$ CIs. Statistical analyses will be undertaken using R statistical software.

\section{Sample size estimation}

Given estimates of our previous exploratory analysis using the Scottish Health Surveys $(31 \%$ using any hormonal contraception, $6.5 \%$ with clinician-diagnosed asthma and 
an OR of $0.68,95 \%$ CI 0.47 to 0.98$),{ }^{15}$ we determined that in order to have $90 \%$ power at an alpha level of 0.05 to detect up to $20 \%$ reduction (risk ratio 0.8 ) in asthma attacks, we will need a sample size of 23700 reproductive age females for use of any hormonal contraception. Furthermore, with $90 \%$ power at an alpha level of 0.05 , we determined that we will need 6000 perimenopausal/ postmenopausal females to detect up to $40 \%$ (risk ratio 1.40) increased risk of asthma attack for use of any HRT. ${ }^{23}$ Currently, the OPCRD database has over 5 million population of patients. We anticipate that about 500000 patients will meet the criteria for inclusion into this study.

\section{Reporting and dissemination plans}

We will report the findings of the study following the recommendations of Strengthening the Reporting of Observational Studies in Epidemiology ${ }^{39}$ and Reporting of studies Conducted using Observational Routinely-collected health Data statements. ${ }^{40}$ All the analysis source codes will be made available at GitHub website (https:// github.com/asthmalhs). The project findings will be presented at national and international scientific meetings and published in international journals. Furthermore, we will capitalise on the dissemination infrastructures of the Asthma UK Centre for Applied Research (eg, the Twitter feed and dynamic website) and the University of Gothenburg to publicise our findings to clinicians, academics, patients and reproductive health channels.

\section{Author affiliations}

${ }^{1}$ Krefting Research Centre, Institute of Medicine, University of Gothenburg, Gothenburg, Sweden

${ }^{2}$ Wallenberg Centre for Molecular and Translational Medicine, Institute of Medicine, University of Gothenburg, Gothenburg, Sweden

${ }^{3}$ Asthma UK Centre for Applied Research, Centre for Medical Informatics, The Usher Institute of Population Health Sciences and Informatics, The University of Edinburgh, Edinburgh, UK

${ }^{4}$ Optimum Patient Care, 5 Coles Lane, Cambridge, UK

${ }^{5}$ Medical Research Council Centre for Reproductive Health, Queen's Medical

Research Institute, University of Edinburgh, Edinburgh, UK

${ }^{6}$ Academic Primary Care, Division of Applied Health Sciences, University of Aberdeen, Aberdeen, UK

${ }^{7}$ Observational and Pragmatic Research Institute, Singapore, Singapore

${ }^{8}$ MRC-Asthma UK Centre in Allergic Mechanisms of Asthma, Division of Asthma,

Allergy and Lung Biology, Guys Hospital, Kings College London, London, UK

Contributors BIN and AS conceived the idea for this work. It was drafted by BIN and was then revised after several rounds of critical comments from CRS, CMH and AS and additional feedback from INS, RP, HC, FA, DR and DBP.

Funding This work was supported by Asthma UK, grant number: AUK-IG-2016346. BIN, INS, CRS and AS were in addition support by the Farr Institute and Asthma UK Centre for Applied Research. BIN acknowledges the support of Knut and Alice Wallenberg Foundation and the Wallenberg Centre for Molecular and Translational Medicine, University of Gothenburg, Sweden.

Competing interests None declared.

Patient consent Not required.

Ethics approval The main ethical issues relate to anonymity, confidentiality, data protection and the linkage of datasets. We have obtained approval (reference number: ADEPT1317) from the Anonymised Data Ethics and Protocol Transparency Committee which grants project-specific approvals for the use of the OPCRD data. Optimum Patient Care has an existing NHS Health Research Authority ethics approval for the use of OPCRD for research (REC Ref: 15/EM/150). All researchers involved in data analysis will have successfully completed the appropriate information governance courses.

Provenance and peer review Not commissioned; externally peer reviewed.

Open access This is an open access article distributed in accordance with the Creative Commons Attribution Non Commercial (CC BY-NC 4.0) license, which permits others to distribute, remix, adapt, build upon this work non-commercially, and license their derivative works on different terms, provided the original work is properly cited, appropriate credit is given, any changes made indicated, and the use is non-commercial. See:@http://creativecommons.org/licenses/by-nc/4.0/.

\section{REFERENCES}

1. Baibergenova A, Thabane L, Akhtar-Danesh N, et al. Sex differences in hospital admissions from emergency departments in asthmatic adults: a population-based study. Ann Allergy Asthma Immunol 2006;96:666-72.

2. Osman M, Hansell AL, Simpson CR, et al. Gender-specific presentations for asthma, allergic rhinitis and eczema in primary care. Prim Care Respir J 2007;16:28-35.

3. Prescott E, Lange P, Vestbo J, et al. Effect of gender on hospital admissions for asthma and prevalence of self-reported asthma: a prospective study based on a sample of the general population. Copenhagen City Heart Study Group. Thorax 1997;52:287-9.

4. Kynyk JA, Mastronarde JG, McCallister JW. Asthma, the sex difference. Curr Opin Pulm Med 2011;17:6-11.

5. Bonds RS, Midoro-Horiuti T. Estrogen effects in allergy and asthma. Curr Opin Allergy Clin Immunol 2013;13:92-9.

6. Triebner K, Johannessen A, Puggini L, et al. Menopause as a predictor of new-onset asthma: A longitudinal Northern European population study. J Allergy Clin Immunol 2016;137:50-7.

7. McCleary N, Nwaru BI, Nurmatov UB, et al. Endogenous and exogenous sex steroid hormones in asthma and allergy in females: A systematic review and meta-analysis. J Allergy Clin Immunol 2018;141:1510-3.

8. Haggerty CL, Ness RB, Kelsey S, et al. The impact of estrogen and progesterone on asthma. Ann Allergy Asthma Immunol 2003;90:284-91.

9. Verthelyi D. Female's heightened immune status: estrogen, T cells, and inducible nitric oxide synthase in the balance. Endocrinology 2006;147:659-61.

10. Fuseini $\mathrm{H}$, Newcomb DC. Mechanisms driving gender differences in asthma. Curr Allergy Asthma Rep 2017;17:19.

11. Newcomb DC, Cephus JY, Boswell MG, et al. Estrogen and progesterone decrease let-7f microRNA expression and increase IL-23/IL-23 receptor signaling and IL-17A production in patients with severe asthma. J Allergy Clin Immunol 2015;136:1025-34.

12. Forbes L, Jarvis D, Burney P. Do hormonal contraceptives influence asthma severity? Eur Respir J 1999;14:1028-33.

13. Jenkins MA, Dharmage SC, Flander LB, et al. Parity and decreased use of oral contraceptives as predictors of asthma in young women. Clin Exp Allergy 2006;36:609-13.

14. Lange P, Parner J, Prescott E, et al. Exogenous female sex steroid hormones and risk of asthma and asthma-like symptoms: a cross sectional study of the general population. Thorax 2001;56:613-6.

15. Nwaru BI, Sheikh A. Hormonal contraceptives and asthma in women of reproductive age: analysis of data from serial national Scottish Health Surveys. J R Soc Med 2015;108:358-71.

16. Scott HA, Gibson PG, Garg ML, et al. Sex hormones and systemic inflammation are modulators of the obese-asthma phenotype. Allergy 2016;71:1037-47.

17. Salam MT, Wenten M, Gilliland FD. Endogenous and exogenous sex steroid hormones and asthma and wheeze in young women. $J$ Allergy Clin Immunol 2006;117:1001-7.

18. Barr RG, Wentowski CC, Grodstein F, et al. Prospective study of postmenopausal hormone use and newly diagnosed asthma and chronic obstructive pulmonary disease. Arch Intern Med 2004;164:379-86.

19. Gómez Real F, Svanes C, Björnsson EH, et al. Hormone replacement therapy, body mass index and asthma in perimenopausal women: a cross sectional survey. Thorax 2006;61:34-40.

20. Jarvis D, Leynaert B. The association of asthma, atopy and lung function with hormone replacement therapy and surgical cessation of menstruation in a population-based sample of English women. Allergy 2008;63:95-102.

21. Romieu I, Fabre A, Fournier A, et al. Postmenopausal hormone therapy and asthma onset in the E3N cohort. Thorax 2010;65:292-7.

22. Tattersfield AE. Is postmenopausal HRT a risk factor for adult-onset asthma? Thorax 2010;65:282-. 
23. Troisi RJ, Speizer FE, Willett WC, et al. Menopause, postmenopausal estrogen preparations, and the risk of adult-onset asthma. A prospective cohort study. Am J Respir Crit Care Med 1995;152:1183-8.

24. Chandler MH, Schuldheisz S, Phillips BA, et al. Premenstrual asthma: the effect of estrogen on symptoms, pulmonary function, and beta 2-receptors. Pharmacotherapy 1997;17:224-34.

25. Matsuo N, Shimoda T, Matsuse $\mathrm{H}$, et al. A case of menstruationassociated asthma: treatment with oral contraceptives. Chest 1999;116:252-3

26. Wei J, Gerlich J, Genuneit J, et al. Hormonal factors and incident asthma and allergic rhinitis during puberty in girls. Ann Allergy Asthma Immunol 2015;115:21-7.

27. Williams T, van Staa T, Puri S, et al. Recent advances in the utility and use of the General Practice Research Database as an example of a UK Primary Care Data resource. Ther Adv Drug Saf 2012;3:89-99.

28. Quint JK, Müllerova H, DiSantostefano RL, et al. Validation of chronic obstructive pulmonary disease recording in the Clinical Practice Research Datalink (CPRD-GOLD). BMJ Open 2014;4:e005540.

29. Souverein PC, Koster ES, Colice G, et al. Inhaled Corticosteroid Adherence Patterns in a Longitudinal Asthma Cohort. J Allergy Clin Immunol Pract 2017;5:448-56.

30. Eisner MD, Katz PP, Yelin EH, et al. Risk factors for hospitalization among adults with asthma: the influence of sociodemographic factors and asthma severity. Respir Res 2001;2:53-60.
31. Charlson ME, Pompei P, Ales KL, et al. A new method of classifying prognostic comorbidity in longitudinal studies: development and validation. J Chronic Dis 1987;40:373-83.

32. Kvaskoff M, Mu F, Terry KL, et al. Endometriosis: a high-risk population for major chronic diseases? Hum Reprod Update 2015;21:500-16.

33. Htet TD, Teede HJ, de Courten B, et al. Asthma in reproductive-aged women with polycystic ovary syndrome and association with obesity. Eur Respir J. In Press. 2017;49:1601334.

34. British Thoracic Society and Scottish Intercollegiate Guidelines Network. British guideline on the management of asthma. Thorax 2016;69:1-192.

35. Rubin DB. Multiple imputation for non-response in surveys: John Wiley, 1987.

36. Austin PC. An Introduction to Propensity Score Methods for Reducing the Effects of Confounding in Observational Studies. Multivariate Behav Res 2011;46:399-424.

37. Lash TL, Fox MP, Fink AK. Applying quantitative bias analysis to epidemiologic data. New York: Springer, 2009.

38. Andersson T, Alfredsson L, Källberg H, et al. Calculating measures of biological interaction. Eur J Epidemiol 2005;20:575-9.

39. von Elm E, Altman DG, Egger M, et al. Strengthening the Reporting of Observational Studies in Epidemiology (STROBE) statement: guidelines for reporting observational studies. BMJ 2007;335:806-8.

40. Benchimol El, Smeeth L, Guttmann A, et al. The REporting of studies Conducted using Observational Routinely-collected health Data (RECORD) statement. PLoS Med 2015;12:e1001885. 